\title{
Bartter syndrome presenting as poor weight gain and dehydration in an infant
}

\author{
N.E.R. Alkhateeb ${ }^{7}$ and Z.T. Gardee ${ }^{7}$
}

\section{Introduction}

Bartter syndrome is a rare metabolic renal tubular disorder characterized by hypokalaemic, hypochloraemic metabolic alkalosis, normal blood pressure, hyper-reninaemia and increased urinary loss of sodium, potassium and chloride [1]. Its estimated prevalence is 1 per million [2].

\section{Case report}

A 14-week-old boy presented with lethargy, poor oral intake and polyuria. There was no history of fever, vomiting or diarrhoea, although he had been previously admitted for dehydration without apparent cause. He was born to consanguineous parents and his mother had a history of previous abortion. Birth weight was $3.5 \mathrm{~kg}$.

Physical examination revealed a lethargic and irritable infant with severe dehydration. He weighed $3 \mathrm{~kg}$ at presentation, which was below the 3rd percentile for age. Other anthropometric measurements were around the 50th percentile (length $58 \mathrm{~cm}$, occipitofrontal circumference $40 \mathrm{~cm}$ ). The results of other examinations were unremarkable.

Biochemical investigation revealed metabolic alkalosis, hypokalaemia and hypochloraemia. Ultrasound and other investigations were normal, although the calcium level was in the upper normal range at presentation, which might have been due to the uses of tonics containing vitamin $\mathrm{D}$; hence he was a case of failure to thrive before the diagnosis of Bartter was considered (Table 1).

It was noticed that he continued to have dehydration despite being on intravenous fluid therapy. At this stage a diagnosis of neonatal Bartter syndrome was considered in view of persistent hypokalaemia and metabolic alkalosis in a baby with failure to thrive and polyuria.
The patient was treated with oral potassium $1.5 \mathrm{meq} / \mathrm{kg}$, spironolactone $3 \mathrm{mg} / \mathrm{kg}$ and indomethacin $2 \mathrm{mg} / \mathrm{kg}$.

He showed adequate weight gain and after 6 months his weight reached $6.5 \mathrm{~kg}$, which was still suboptimal. Consistently normal levels of serum electrolytes were found (Table 1).

\section{Discussion}

Bartter syndrome, originally described by Bartter et al. in 1962 [3], is a primary tubulopathy that present with failure to thrive and is associated with a characteristic biochemical abnormalities such as hypokalaemia, hypochloraemia, metabolic alkalosis, increased urinary excretion of chloride and hyperreninaemia. It presents in infancy, childhood but the diagnosis can be delayed till adulthood [4].

Hypokalaemic, hypochloraemic metabolic alkalosis suggests the

\begin{tabular}{|c|c|c|c|}
\hline \multirow[t]{2}{*}{ Parameter } & \multicolumn{3}{|c|}{ Values } \\
\hline & At presentation & After 1 month & After 6 months \\
\hline Serum sodium (mEq/L) & 135 & 135 & 136 \\
\hline Serum potassium (mEq/L) & 2.5 & 3.7 & 4.4 \\
\hline Serum chloride (mEq/L) & 76.8 & 87.0 & 106.0 \\
\hline Serum magnesium (mEq/L) & 2.8 & - & 2.2 \\
\hline Serum calcium (mEq/L) & 10.7 & - & 9.3 \\
\hline Blood pH & 7.59 & - & - \\
\hline Bicarbonate (mEq/L) & 47.2 & - & - \\
\hline Blood urea (mg/dL) & 27 & - & 34 \\
\hline Serum creatinine (mg/dL) & 0.8 & - & 0.5 \\
\hline
\end{tabular}


possibility of primary or secondary hyperaldosteronism. Of the former, primary mineralocorticoid excess was excluded by the absence of hypertension and high or normal sodium levels [3]. In the latter, when no cause can be identified (e.g. vomiting, diarrhoea, abuse of diuretics or laxatives), the conditions to be differentiated are Bartter and Gitelman syndrome. Classic and neonatal Bartter syndrome have similar presenting symptoms but different presentation ages, Gitelman syndrome is found in late childhood or adolescence and has the classic hallmark finding of hypomagnesaemia, which differentiates it from classic and neonatal variants [5].

Failure to thrive in an infant has multiple etiologies and at times is the only manifestation of underlying serious diseases such as Bartter syndrome, in which the majority of patients present with failure to thrive, vomiting and constipation during the first 2 years of life [6].

Therapeutic efforts should be directed to correct dehydration and electrolyte imbalance. Administration of indomethacin after 6-12 weeks of life $1-5 \mathrm{mg} / \mathrm{kg} /$ day is most frequently used and well tolerated [3]. Remarkable clinical and biochemical improvement was reported in patients treated with indomethacin [7]. As it is an autosomal recessive disorder, genetic counselling should be offered to couple whose previous siblings have suffered from the disorder [4]. With early diagnosis and proper treatment Bartter syndrome has a good prognosis, but failure to identify it can lead to renal failure [8].

This syndrome is reported because of its rarity-to our information this is the first reported in Iraq-and to alert paediatricians in the region to its neonatal variant.

\section{References}

1. Kumar PS et al. Neonatal Bartter syndrome. Indian Pediatrics, 2006, 43:735-737.

2. Ji W et al. Rare independent mutations in renal salt handling genes contribute to blood pressure variation. Nature Genetics, 2008, 40:592.

3. Contractor CP et al. Bartter's syndrome: a neonatal presentation. Journal of Postgraduate Medicine, 1992, 38:41-43.

4. Muranjan $\mathrm{MN}$ et al. Neonatal Bartter syndrome. Indian Journal of Pediatrics, 2002, 69:105-107.

5. Muthukrishnan J et al. Inherited renal tubular defect with hypokalemia. Saudi Journal of Kidney Diseases and Transplantation, 2009, 20:294-297.
6. Allapathi $\mathrm{D}$ et al. Severe failure to thrive in infant. Clinical Pediatrics, 2008, 47:698-700.

7. Abdel-al YK et al. Bartter's syndrome in Arabic children: review of 13 cases. Pediatrics International, 1999, 41:299-303.

8. Heffernan A et al. Bartter syndrome presenting as poor weight gain and abdominal mass in an infant. Fetal and Pediatric Pathology, 2008, 27:232-243. 